\title{
Terahertz time-domain spectroscopy of astrophysical ice analogs: A pilot study
}

\author{
$\underline{\text { A. A. Gavdush }}{ }^{1,2}$, B. M. Giuliano ${ }^{3}$, B. Müller ${ }^{3}$, G. A. Komandin ${ }^{1}$, \\ M. E. Palumbo ${ }^{4}$, G. A. Baratta ${ }^{4}$, C. Sciré $^{4}$, \\ S. O. Yurchenko ${ }^{2}$, K. I. Zaytsev ${ }^{1,2}$, A. V. Ivlev ${ }^{3}$, and P. Caselli ${ }^{3}$ \\ ${ }^{1}$ Prokhorov General Physics Institute of the Russian Academy of Sciences, Moscow, Russia, arsenii.a.gavdush@gmail.com \\ ${ }^{2}$ Bauman Moscow State Technical University, Moscow, Russia \\ ${ }^{3}$ Max-Planck-Institute für extraterrestrische Physik, Garching bei München, München, Germany \\ ${ }^{4}$ Osservatorio Astrofisico di Catania, Catania, Italy
}

Ices are one of the most common kinds of dense matter in the Universe. There are more than 180 various molecular astrophysical ices, including $\mathrm{H} 2 \mathrm{O}, \mathrm{CO}$, $\mathrm{CO} 2, \mathrm{CH} 3 \mathrm{OH}$, etc. [1]. Surface chemical processes on icy mantles of dust particles play an important role in formation of new molecules, including biologically relevant organic species [2,3]. Therefore, thorough studies of the ice properties may shed light to such astrophysical problem, as understanding the origin of the volatiles and organics - the building blocks of life - in space and then on the early Earth.

Analysis of astronomical observations in different ranges of electromagnetic spectrum is the main tool for experimental studies of ices in the Universe. These observations employ mostly a mid-infrared (IR) range of wavelengths $[1,4]$, but also cover far-IR and terahertz $(\mathrm{THz})$ ranges, provided by such space telescopes, as Hershel [5] and SOFIA [6]. A cornerstone of proper interpretation of these observations is their comparison to IR and $\mathrm{THz}$ spectra of astrophysical ice analogs obtained in laboratory conditions. Recent works were mainly dedicated to the analysis in midIR frequency region [1], with only a few works concerned on $\mathrm{THz}$ spectroscopy [7,8]. However, in spite of its importance, the presently available data related to spectroscopy in IR and THz range are still incomplete or even have not been performed for a lot of ice compounds, to our knowledge.

In the present work, we apply previously developed method [9-13] for reconstruction of both real and imaginary parts of dielectric permittivity, using the THz time-domain spectroscopy (TDS). This technique allows us to measure optical properties of astrophysical ice analogs in a broad range of wavelengths, that is required for detailed study of radiative transfer in ice mantles of astrophysical dust particles. We developed an experimental setup allowing simultaneous growth and THz TDS of thin ice films of different molecular compositions.

The experimental setup for the spectroscopic measurements is composed closed-cycle He cryostat and $\mathrm{THz}$ time-domain pulsed spectrometer (TPS). The cryostat is mounted on a motor controlled translational stage to tune its position with respect to the $\mathrm{THz}$ beam. The cryocooler vacuum chamber is equipped with four ports for optical access and for the gas inlet. Optical windows and a substrate for the ice growing are made of high-resistivity float-zone silicon (HRFZ$\mathrm{Si}$ ) with different thickness, the smallest one for the substrate, in order to suppress multiple resonances in the $\mathrm{THz}$ spectra. The pumping station that operates with gas mixture for ice sample deposition is composed of a turbomolecular pump combined with a backing rotary pump. The THz TPS used for the present work has been basically purchased from the Batop $\mathrm{GmbH}$, and it is coupled with two photoconductive antennas made of low-temperature grown gallium arsenide and a femtosecond laser with a pulse repetition rate of $100 \mathrm{MHz}$ and an average input power of $65 \mathrm{~mW}$. Further technical details on an experimental setup one can find in the upcoming work at Astronomy \& Astrophysics journal [11].

Growth of the ices on the substrate is organized using standard technique using vapor deposition on the cold substrate in the vacuum chamber. To attain ice thickness of a mm order, fast deposition regime is employed. The first experiments were performed with ice of $\mathrm{CO}$, deposited at $14 \mathrm{~K}$ in a several deposition regimes, with 4, 5 and 6 min deposition steps, and up to the total 30 min deposition time.

The reconstruction procedure of the optical properties of ice layers is organized in the same manner as in previous works [9-13]. The signal propagated through the empty vacuum chamber at $14 \mathrm{~K}$ was used as the reference one for analysis of the sample signal propagated through the chamber with grown ice layers on the substrate. Then, the time-domain signals (reference ballistic and sample satellite pulses) were analyzed to have initial estimations of ice layers' thicknesses and a real part of the sample refractive index, to enhance the accuracy of data post processing. Reliability of the employed approach is confirmed by independent measurements of the sample thickness [15], and observed linear increase of the total initial thickness of ices in different deposition regimes. In result [14], the optical properties of $\mathrm{CO}$ ice were measured in the range of $0.3-2.0 \mathrm{THz}$, with a clearly expressed Lorentz-like peak centered near 1.5 $\mathrm{THz}$, which stands in a good agreement with previous studies $[16,17]$.

This work was partly supported by the Russian Foundation for Basic Research (RFBR), Project \#1832-00816.

\section{References}

1. Boogert, A. C. A., Gerakines, P. A., and Whittet, D. C. B. Observations of the icy universe // Annual Review of Astronomy and Astrophysics 2015. Vol. 53, P. 541-581.

2. Öberg K. I. Photochemistry and Astrochemistry: Photochemical Pathways to Interstellar Complex Organic 
Molecules // Chemical Reviews 2016. V. 116, No 17 P. 9631-9663.

3. McGuire, B. A., Carroll, P. B., Loomis, R. A.., Finneran, I. A., Jewell, P. R., Remijan, A. J., Blake, G. A. Discovery of the interstellar chiral molecule propylene oxide (CH3CHCH2O) // Science 2016. V. 352, No. 6292. P. 1449-1452.

4. van Dishoeck, E. F. Astrochemistry of dust, ice and gas: introduction and overview // Faraday Discuss. 2014. V. 168, P. 9-47.

5. Doyle, D., Pilbratt, G. and Tauber, J. The Herschel and Planck Space Telescopes // Proceedings of the IEEE 2009. V. 97, No. 8. P. 1403-1411.

6 Gehrz, R. D., Becklin, E. E., de Pater, I., Lester, D. F., Roellig, T. L. and Woodward, C. E. A new window on the cosmos: The Stratospheric Observatory for Infrared Astronomy (SOFIA) // Advances in Space Research 2009. V. 44, No. 4. P. 413-432.

7. Allodi, M. A., Ioppolo, S., Kelley, M. J., McGuire, B. A., and Blake, G. A. The structure and dynamics of carbon dioxide and water containing ices investigated via $\mathrm{THz}$ and mid-IR spectroscopy // Physical Chemistry Chemical Physics 2014. V. 16, No. 8. P. 3442-3455.

8. McGuire, B. A., Ioppolo, S., Allodi, M. A., and Blake, $G$. $A$. $\mathrm{THz}$ time-domain spectroscopy of mixed $\mathrm{CO} 2-$ $\mathrm{CH} 3 \mathrm{OH}$ interstellar ice analogs // Physical Chemistry Chemical Physics 2016. V. 18, No. 30. P. 20199-20207.

9 Zaytsev, K. I., Gavdush, A. A., Lebedev, S. P., Karasik, V. E., and Yurchenko S. O. A Method of Studying Spectral Optical Characteristics of a Homogeneous Medium by Means of Terahertz Time-Domain Spectroscopy // Optics and Spectroscopy 2015. V. 118, No. 4. P. 552-562.

10. Zaytsev, K. I., Gavdush, A. A., Chernomyrdin, N. $V$., and Yurchenko, S. O. Highly Accurate In Vivo Terahertz Spectroscopy of Healthy Skin: Variation of Refractive Index and Absorption Coefficient along the Human Body //
IEEE Transactions on Terahertz Science and Technology 2015. V. 5, No. 5. P. 817-827.

11. Yakovlev, E. V., Zaytsev, K. I., Dolganova, I. N., Yurchenko, S. O. Non-Destructive Evaluation of Polymer Composite Materials at the Manufacturing Stage Using Terahertz Pulsed Spectroscopy // IEEE Transactions on Terahertz Science and Technology 2015. V. 5, No. 5. P. 810 -816 .

12. Zaytsev, K. I., Kudrin, K. G., Karasik, V. E., Reshetov, I. V., Yurchenko S. O. In vivo terahertz spectroscopy of pigmentary skin nevi: Pilot study of non-invasive early diagnosis of dysplasia // Applied Physics Letters 2015. V. 106, No. 5 P. 053702.

13. Zaytsev, K. I., Gavdush, A. A., Karasik, V. E., Alekhnovich, V. I., Nosov, P. A., Lazarev, V. A., Reshetov, I. V., Yurchenko, S. O. Accuracy of sample material parameters reconstruction using terahertz pulsed spectroscopy // Journal of Applied Physics 2014. V. 115, P. 193105.

14. Giuliano, B. M., Gavdush, A. A., Müller, B., Zaytsev, K. I., Palumbo, M. E., Baratta, G. A., Sciré, C., Komandin, G. A., Yurchenko, S. O., Ivlev, A. V., and Caselli, $P$. Broadband spectroscopy of astrophysical ice analogs. I. Direct measurement of complex refractive index using terahertz pulsed spectroscopy // Astronomy \& Astrophysics manuscript.

15. Baratta, G. A., Palumbo, M. E. Infrared optical constants of $\mathrm{CO}$ and $\mathrm{CO} 2$ thin icy films // J. Opt. Soc. Am. A 1998. V. 15, No. 12. P. 30763085 .

16. Anderson, A., Leroi, G. E. Far-Infrared Spectra of Crystalline Nitrogen and Carbon Monoxide // The Journal of Chem. Phys. 1966. V. 45, P. 4359.

17. Ron, A., Schnepp, $O$. Lattice Vibrations of the Solids $\mathrm{N}_{2}, \mathrm{CO}_{2}$, and $\mathrm{CO} / /$ The Journal of Chem. Phys. 1967. V. 46, No. 10 P. 3991-3998. 